\title{
Correlation between Central Venous Pressure and the Diameter of Inferior Vena Cava by using Ultrasonography for the Assessment of the Fluid Status in Intensive Care Unit Patients

\author{
Mostafa Ibrahim Mostafa Shalaby*; Hussein Montasser Roshdy**; Wael Mohamed Elmahdy*; Anas \\ Ezzat Fathy El Mezayen* \\ Departments of Anesthesia and Intensive Care Unit* and Diagnostic Radiology**, \\ Faculty of Medicine - Al Azhar University
} \\ Corresponding Author: Anas Ezzat Fathy El Mezayen, Mobile: 01009836758; Email: anasezzat1990@ gmail.com
}

\begin{abstract}
Background: decisions regarding fluid therapy, whether in the operating theatre, intensive care unit, emergency department, are among the most challenging and important tasks that clinicians face on a daily basis. Specifically, almost all clinicians would agree that both hypovolaemia and volume overload increase the morbidity and mortality of patients. The therapeutic goal of fluid administration is to increase preload, or the stressed venous volume, leading to an increased stroke volume and cardiac output. However, studies of patients with acute illness or hypotensive patients in the intensive care unit consistently demonstrate that approximately $50 \%$ of fluid boluses fail to achieve the intended effect of increasing cardiac output. Aim of the Work: this study was done to evaluate the correlation between central venous pressure (CVP) measurements and ultrasound measurements of the inferior vena cava diameter, and collapsibility index. The secondary aim was to evaluate the value of ultrasound as a noninvasive tool in assessment of intravascular volume status and fluid responsiveness in critically ill intensive care unit patients. Patients and Methods: after obtaining the approval of the Al-Azhar University Ethical Committee and written informed consent, 50 patients aged 30-60 years of either sex, ASA I-III admitted in the ICU of Al-Azhar teaching hospitals who had a functioning central venous catheter inserted for any clinical indication, were involved in this single blinded correlational study. Hemodynamic parameters were monitored continuously including heart rate and non-invasive mean arterial blood pressure. CVP measurements were taken with the patient in the supine position. Clinical assessment was done for signs of hypovolemia like hypotension, tachycardia, prolongation of capillary refill: $>3$ seconds, acidosis, increased serum lactate more than $2 \mathrm{mmol} / \mathrm{L}$ or loss of skin turgor. Results: in our study, there was a significant correlation between CVP and the two studied ultrasound parameters, IVC CI and IVCdmax. Analysis of the receiver operating characteristic curve ROC showed that inferior vena cava collapsibility index (IVC CI) had the most favorable performance of the two ultrasound parameters in predicting CVP $<10 \mathrm{~cm} \mathrm{H2O}$. As regards prediction of fluid responsiveness, analysis of the ROC showed a better diagnostic accuracy of IVC collapsibility index and IVC diameter for predicting fluid responsiveness. Conclusion: ultrasound of the inferior vena cava may be used as a feasible non-invasive, rapid and simple adjuvant method to assess the intravascular volume and guide fluid responsiveness in critically ill intensive care unit patients, inferior vena cava collapsibility index may be used to predict low central venous pressure and predict fluid responsiveness.
\end{abstract}

Keywords: Central venous pressure, diameter of inferior vena cava

\section{INTRODUCTION}

Fluid management in the intensive care plays a vital role in the outcome of the patient. Hypovolemia with inappropriate use of vasopressors to maintain the blood pressure reduces the organ perfusion leading to ischemia. On the other hand fluid overload causes cellular swelling and congestion of lungs thereby increasing morbidity and mortality. ${ }^{(1)}$ Central venous catheters have a wide variety of uses such as hemodynamic monitoring, drug administration, total parenteral nutrition, trans-venous pacemaker placement, pulmonary artery catheterization, etcetera. The central venous pressure is a static measure of volume. ${ }^{(2)}$ The method has been followed widely to assess the volume status and thereby treating the patient accordingly. Insertion of central venous catheter is contraindicated in certain situation as any coagulation disorders, infection over the insertion site etc. There have been reports of many complications with a central venous catheter e.g. infections, accidental arterial puncture, hematoma, hemothorax, pneumothorax, air embolism, dysrhythmias. ${ }^{\text {(3) Recently the ultrasound guided }}$ measurement of the IVC diameter and its changes with respiration have been used to calculate the fluid status of a patient. It is a safe technique and it is also relatively cheap. It can be used as an alternative to central venous catheterization to assess the volume status of patients. It is a dynamic measure of intravascular volume status, as it reflects the volume changes that take place with respiration. ${ }^{(4)}$ The IVC adjusts to the body's volume status by changing its diameter depending on the total body fluid volume. The caval opening increases in size during 
inspiration, which encourages venous return of blood to the heart through the IVC due to the negative intrathoracic pressure. This results in the collapse of the IVC. During expiration the reverse happens, where due to the positive intra-thoracic pressure the pressure gradient decreases causing a distension of the IVC. ${ }^{(4)}$ Different techniques are employed for this purpose which include physical examination, central venous pressure (CVP) measurement, biochemical markers, estimate of the vascular pedicle width, pulmonary artery catheters, sonographic inferior vena cava (IVC) diameter assessment and various catheter devices. ${ }^{(5)}$ Bedside ultrasonography is readily available in intensive care setups. It is safe, cheap and non-invasive. Ultrasound of inferior vena cava (IVC) is a tool that can provide a rapid and non-invasive means of gauging preload and the need for fluid resuscitation. ${ }^{(6)}$

\section{AIM OF THE WORK}

This study was done to evaluate the correlation between CVP measurements and ultrasound measurements of the inferior vena cava diameter, and collapsibility index. The secondary aim was to evaluate the value of ultrasound as a noninvasive tool in assessment of intravascular volume status and fluid responsiveness in critically ill intensive care unit patients.

\section{PATIENTS AND METHODS}

After obtaining the approval of the AlAzhar University Ethical Committee and written informed consent, 50 patients of both sex admitted in the ICU of Al-Azhar university hospitals who had a functioning central venous catheter inserted for any clinical indication, were involved in this single blinded Correlational study. Inclusion criteria: Both genders, age: 30-60 years, not intubated, not ventilated, hypotensive patients (mean arterial blood pressure less than $65 \mathrm{mmHg}$ ), ASA I-III. Exclusion Criteria: patients with sever orthopnea, morbid obese B.M.I. above $50 \mathrm{~kg} / \mathrm{m}^{2}$, suspected or diagnosed raised intra-abdominal or intra-thoracic pressures as known pregnancy, portal hypertension, or mediastinal mass, intracerebral hemorrhage or increased ICP, valvar heart disease (moderate-to-severe tricuspid regurgitation), atrial fibrillation, inability to obtain adequate quality US images (e.g. abdominal distension).

Methods: Assessment: All patients included in the study were assessed by: Patients' demographic data: age, sex, body weight and height: 1. Detailed medical, surgical history, 2. Complete clinical examination, 3. Routine laboratory investigations and serum lactate, 4. Chest x-ray, 5. Standard 12 lead electrocardiogram (ECG).

Monitoring: Standard monitoring was applied, including non-invasive arterial blood pressure, electrocardiography and pulse oximetry using the multichannel monitor. Technique: Patients were asked to lie supine, with the bed flat. The basic monitors (ECG, NIBP and SpO2) were attached. The vital parameters were stable for the patients during the study. We asked our patients to lie supine and use ultrasound machine to take ultrasound images of IVC diameter. A portable ultrasound machine with $17 \mathrm{~mm}$ curved probe and cardiac transducer for IVC imaging 1-5 MHz, $21 \mathrm{~mm}$ phase array was used. Subxiphoid approach was used to visualize IVC. We measured maximum anteroposterior diameter of IVC at the end of inspiration. Hypovolemic IVC (H-IVC) was defined by diameter less than 2 centimeters and normovolemic IVC (N-IVC) was defined as IVC diameter equal or more than 2 centimeters. All the readings were taken by team of two intensive care physicians and assistant nurse. This team sought specialized training in use of bed side ultrasonography by specialist radiologists. Patients were grouped on the basis for decision making in fluid management. Data have been collected before start of fluid therapy and after fluid challenge test and 3 and 6 hours after the start of fluid management, analyzed using SPSS version 18. Measurements: $\mathbf{1}$. Hemodynamic parameters were monitored continuously: heart rate (HR): beats/min, noninvasive mean arterial blood pressure (MABP): $\mathrm{mmHg}$. 2. Central venous pressure (CVP): $\mathrm{cm}$ $\mathrm{H}_{2} \mathrm{O}$. The transducer used to measure the central venous pressure was fixed at the level of the patient's phlebostatic axis, the level at which the fourth intercostal space meets the midaxillary line. The transducer was connected to the monitor using its cable. After zeroing, the transducer was left open to the central venous catheter. CVP waveform displayed on the monitor with the average central venous pressure measured in $\mathrm{mm} \mathrm{Hg}$. This provided a means to measure the CVP continuously. This measurement was taken by another anesthesiologist and the principal investigator was blinded to this value. 3 . Clinical assessment for signs of hypovolemia: ${ }^{(7)}$ Hypotension: defined as mean arterial blood pressure less than $65 \mathrm{mmHg}$ in two consecutively reading with 
interval two minutes by using non-invasive technique. Tachycardia: defined as heart rate more than $20 \%$ from the baseline reading or more than 100 beat/minute, prolongation of capillary refill: >3 seconds, acidosis, increased serum lactate more than $2 \mathrm{mmol} / \mathrm{L}$. 4. Ultrasound measurements including: Maximum IVC diameter at end expiration $\left(\mathrm{IVCd}_{\max }\right)$ : $\mathrm{cm}$. To measure the IVC diameter, a curvilinear probe of sonosite turbo ultrasound machine was placed longitudinally in the subxiphoid region with the probe marker to the patient's head, to visualize the confluence of the hepatic veins draining the IVC. We may need to move the probe $1-2 \mathrm{~cm}$ to the patient's right and then tilt it slightly towards the heart. The internal anterior posterior [AP] diameter of the IVC just caudal to the confluence of the hepatic veins in the longitudinal plane was measured. ${ }^{(8)}$ Minimum IVC diameter at end inspiration $\left(\mathrm{IVCd}_{\min }\right): \mathrm{cm}$. The IVC collapsibility index (IVC CI):\%. The difference between the maximum and minimum IVC diameters divided by the maximum IVC diameter, expressed as a percentage $\left(\left[\mathrm{IVCd}_{\max }-\mathrm{IVCd}_{\min }\right] / \mathrm{IVCd}_{\max } \times\right.$ $100 \%)$. (9) The previous measurements were recorded in a spontaneously breathing patient at the following timings: Baseline measurement before fluid challenge test, immediately after fluid challenge test, 3 hours after fluid challenge test, 6 hours after challenge test. And also during the following events: CVP less than $10 \mathrm{~cm} \mathrm{H}_{2} \mathrm{O}$ or any of the previously mentioned signs of hypovolemia was observed, after passive leg raising 45 degrees: In case of signs of hypovolemia or CVP less than $10 \mathrm{~cm}$ $\mathrm{H}_{2} \mathrm{O}$, passive leg raising test was done to exclude other causes like cardiac dysfunction or volume intolerance. Passive leg raising test was thus performed with CVP as a safety limit. Automatic bed elevation or wedge pillow was used while at the same time placing the patient in the supine from a $45^{\circ}$ semi-recumbent position. If PLR induced an increase of CVP of $<5 \mathrm{~cm} \mathrm{H}_{2} \mathrm{O}$, fluid bolus was given. If PLR caused a high increase of CVP of $>5 \mathrm{~cm} \mathrm{H}_{2} \mathrm{O}$ (according to the "2-5" rules for CVP), further infusion of fluid was considered unsafe as it suggests the possibility of cardiac dysfunction and volume intolerance and the patient was excluded from the study. ${ }^{(10)}$ After administration of $6 \%$ hydroxyethyl starch (HES, 130/0.4) fluid bolus of $7 \mathrm{ml} / \mathrm{kg}$ over 30 minutes, in case of signs of hypovolemia or CVP less than $10 \mathrm{~cm} \mathrm{H}_{2} \mathrm{O}$. A patient was defined as fluid responder if the mean arterial pressure increased by at least $20 \%$ as compared with pre-fluid challenge values. Several studies also used MABP as a guide for fluid administration, and defined fluid responsiveness by the increase in MABP. ${ }^{(11)} \mathrm{We}$ performed outcome measurements at 30 minutes after the end of fluid challenge to avoid misclassification of transient effects as clinically relevant effects. At this time, the volume effect of hydroxyethyl starch was fully maintained. (11) Hydroxyethyl starch infusion was repeated as long as there was a positive response until improvement of the pre-fluid challenge sign of hypovolemia. Hydroxyethyl starch infusion was stopped if one of the following conditions occurred: Central venous pressure increased to a value greater than $15 \mathrm{~cm} \mathrm{H}_{2} \mathrm{O}$, mean arterial pressure increased to a value greater than $75 \mathrm{~mm} \mathrm{Hg}$. 5 . Laboratory measures: Serum lactate $\mathrm{mmol} / \mathrm{L}$. Statistical analysis of the data: Data were fed to the computer and analyzed using IBM SPSS software package version 20.0. (Armonk, NY: IBM Corp) Qualitative data were described using number and percent. The Kolmogorov-Smirnov test was used to verify the normality of distribution. Quantitative data were described using range (minimum and maximum), mean, standard deviation and median. Significance of the obtained results was judged at the $5 \%$ level. Mean and standard deviation (SD) were calculated for quantitative variables. The comparison between more than two groups with parametric distribution were done by using One Way Analysis of Variance (ANOVA). Pearson correlation coefficient was calculated to study relationship between CVP and IVC diameter. A p-value $<.05$ was considered as significant.

\section{RESULTS}

Table (1): Mean changes in heart rate (HR) during the studied periods

\begin{tabular}{|l|c|c|c|c|c||}
\hline \multirow{2}{*}{} & \multirow{2}{*}{ Baseline } & \multicolumn{3}{|c|}{ After } & \multicolumn{2}{c||}{ After } \\
\cline { 2 - 6 } & & $\begin{array}{c}\text { PLR } \\
\text { test }\end{array}$ & $\begin{array}{c}\text { Bolus fluid } \\
\text { administration }\end{array}$ & 3 hours & 6 hours \\
\hline \hline Min. & 52 & 58 & 68 & 65 & 60 \\
\hline Max. & 109 & 95 & 92 & 95 & 96 \\
\hline Mean & 82.56 & 81.87 & 81.55 & 79.89 & 78.28 \\
\hline \pm SD. & 10.78 & 7.74 & 7.17 & 8.42 & 8.63 \\
\hline $\mathrm{p}_{1}$ & & & & $<0.001^{*}$ & $<0.001^{*}$ \\
\hline
\end{tabular}

$\mathrm{p}: \mathrm{p}$ value for $\mathrm{F}$ test (ANOVA) with repeated measures for comparing between in presence of signs of hypovolemia with after PLR test and Bolus fluid administration

*: Statistically significant at $\mathrm{p} \leq 0.05$ 
Table (2): Mean changes in mean arterial blood pressure (MABP) during the studied periods.

\begin{tabular}{|c|c|c|c|c|c|}
\hline & \multicolumn{5}{|c|}{ MABP (mmHg) } \\
\hline & \multirow[b]{2}{*}{ Baseline } & \multicolumn{2}{|r|}{ After } & \multicolumn{2}{|c|}{ After } \\
\hline & & $\begin{array}{c}\text { PLR } \\
\text { test }\end{array}$ & $\begin{array}{c}\text { Bolus fluid } \\
\text { administration }\end{array}$ & $\begin{array}{c}3 \\
\text { hours }\end{array}$ & $\begin{array}{c}6 \\
\text { hours }\end{array}$ \\
\hline Min. & 48 & 58 & 63 & 58 & 55 \\
\hline Max. & 92 & 88 & 88 & 87 & 89 \\
\hline Mean & 72.26 & 69.16 & 72.52 & 75.89 & 74.58 \\
\hline \pm SD. & 10.80 & 6.09 & 4.87 & 6.64 & 8.41 \\
\hline $\mathrm{p}_{1}$ & & & & $<0.001^{*}$ & $<0.001^{\prime \prime}$ \\
\hline
\end{tabular}

$p: p$ value for $F$ test (ANOVA) with repeated measures for comparing between the presence of signs of hypovolemia with after PLR test and Bolus fluid administration

*: Statistically significant at $\mathrm{p} \leq 0.05$

Table (3): Mean changes in central venous pressure (CVP) during the studied periods.

\begin{tabular}{|c|c|c|c|c|c|}
\hline & \multicolumn{5}{|c|}{$\overline{\mathrm{CVP}\left(\mathrm{cmH}_{2} \mathrm{O}\right)}$} \\
\hline & \multirow[b]{2}{*}{ Baseline } & \multicolumn{2}{|r|}{ After } & \multicolumn{2}{|c|}{ After } \\
\hline & & $\begin{array}{c}\text { PLR } \\
\text { test }\end{array}$ & $\begin{array}{c}\text { Bolus fluid } \\
\text { administration }\end{array}$ & 3 hours & 6 hours \\
\hline Min. & $\overline{0}$ & 4 & $\overline{6} 6$ & 3 & $\overline{1} 1$ \\
\hline Max. & 13 & 10 & 11 & 13 & 13 \\
\hline Mean & 8.06 & 7.35 & 8.24 & 9.33 & 9.11 \\
\hline \pm SD. & 2.96 & 1.53 & 1.41 & 2.08 & 2.45 \\
\hline $\mathrm{p}_{1}$ & & & & $<0.001^{*}$ & $<0.001^{*}$ \\
\hline
\end{tabular}

$\mathrm{p}: \mathrm{p}$ value for $\mathrm{F}$ test (ANOVA) with repeated measures for comparing between the presence of signs of hypovolemia with after PLR test and Bolus fluid administration

*: Statistically significant at $\mathrm{p} \leq 0.05$

Table (4): Mean changes in inferior vena cava maximum diameter $\left(\mathrm{IVCd}_{\max }\right)$ during the studied periods.

\begin{tabular}{|c|c|c|c|c|c|}
\hline \multirow{3}{*}{$\mathrm{IVCd}_{\max }$} & \multicolumn{5}{|c|}{$\mathrm{IVCd}_{\max }(\mathrm{cm})$} \\
\hline & \multirow[b]{2}{*}{ Baseline } & \multicolumn{2}{|r|}{ After } & \multicolumn{2}{|c|}{ After } \\
\hline & & $\begin{array}{c}\text { PLR } \\
\text { test }\end{array}$ & $\begin{array}{c}\text { Bolus fluid } \\
\text { administration }\end{array}$ & \begin{tabular}{|c|}
3 \\
hours
\end{tabular} & \begin{tabular}{|c|}
6 \\
hours
\end{tabular} \\
\hline Min. & 1.24 & 1.22 & 1.36 & 1.36 & 1.38 \\
\hline Max. & 2.3 & 2.32 & 2.31 & 2.42 & 2.52 \\
\hline Mean & 1.82 & 1.72 & 1.78 & 1.84 & 1.87 \\
\hline$\pm \mathrm{SD}$ & 0.27 & 0.24 & 0.24 & 0.24 & 0.26 \\
\hline $\mathrm{p}_{1}$ & & & & $<0.001$ & $<0.001$ \\
\hline
\end{tabular}

$p$ : $p$ value for $F$ test (ANOVA) with repeated measures for comparing between the presence of signs of hypovolemia with after PLR test and Bolus fluid administration

*: Statistically significant at $\mathrm{p} \leq 0.05$
Table (5): Mean changes in inferior vena cava collapsibility index (IVC CI) during the studied periods.

\begin{tabular}{|c|c|c|c|c|c|}
\hline & \multicolumn{5}{|c|}{ IVC CI \% } \\
\hline & \multirow[b]{2}{*}{ Baseline } & \multicolumn{2}{|r|}{ After } & \multicolumn{2}{|c|}{ After } \\
\hline & & $\begin{array}{c}\text { PLR } \\
\text { test }\end{array}$ & $\begin{array}{c}\text { Bolus fluid } \\
\text { administration }\end{array}$ & \begin{tabular}{|c|}
3 \\
hours
\end{tabular} & 6 hours \\
\hline Min. & 19.47 & 17.65 & 14.49 & 18.92 & 16.87 \\
\hline Max. & 67.42 & 52.24 & 47.79 & 53.25 & 62.8 \\
\hline Mean & 36.80 & 37.88 & 34.48 & 32.73 & 35.08 \\
\hline \pm SD. & 11.30 & 7.68 & 8.25 & 9.06 & 10.32 \\
\hline $\mathrm{p}_{1}$ & & & & $<0.00^{*}$ & $<0.001^{*}$ \\
\hline
\end{tabular}

$\mathrm{p}: \mathrm{p}$ value for $\mathrm{F}$ test (ANOVA) with repeated measures for comparing between the presence of signs of hypovolemia with after PLR test and Bolus fluid administration

*: Statistically significant at $\mathrm{p} \leq 0.05$

Table (6): Correlation between CVP and ultrasound measurements of $\mathrm{IVCd}_{\max }$, IVC CI, at baseline.

\begin{tabular}{|c|c|c|}
\hline \multirow{2}{*}{ Baseline } & \multicolumn{2}{|c|}{ CVP } \\
\cline { 2 - 3 } & $\mathbf{r}$ & $\mathbf{p}$ \\
\hline \hline IVCd $_{\max }$ & $0.547^{*}$ & $<0.001$ \\
\hline IVC CI $^{*}$ & $-0.788^{*}$ & $<0.001$ \\
\hline
\end{tabular}

r: Pearson coefficient

*: Statistically significant at $\mathrm{p} \leq 0.05$

Table (7): Test Characteristics of the four ultrasound parameters in predicting the baseline CVP $<10 \mathrm{cmH}_{2} \mathrm{O}$.

\begin{tabular}{|c|c|c|c|c|c|c|c|c|c|}
\hline \multirow{2}{*}{ Baseline } & \multirow{2}{*}{ AUC } & \multirow[b]{2}{*}{ p } & $95 \%$ & C.I & \multirow{2}{*}{$\begin{array}{l}\text { Cut } \\
\text { off }\end{array}$} & \multirow{2}{*}{\multicolumn{2}{|c|}{\begin{tabular}{c|c} 
Sensi- & Speci- \\
tivity & ficity
\end{tabular}}} & & \multirow{2}{*}{ NPV } \\
\hline & & & "LL & 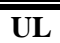 & & & & & \\
\hline IVC & .78 & $0.012^{*}$ & 0.622 & 0.905 & $\leq 1.73$ & 71.40 & 75.60 & & \\
\hline IVC & 996 & $000 \%$ & 787 & 0980 & $>33.4$ & 79.80 & 96.6 & & \\
\hline
\end{tabular}

Table (8): Test Characteristics of the four ultrasound parameters in predicting $\mathrm{CVP}<10 \mathrm{~cm}$ $\mathrm{H}_{2} \mathrm{O}$ in all the study periods.

\begin{tabular}{|c|c|c|c|c|c|c|c|c|c|}
\hline & \multirow{2}{*}{ AUC } & \multirow{2}{*}{$\mathbf{p}$} & \multicolumn{2}{|c|}{\begin{tabular}{|l|}
$55 \%$ C.I \\
\end{tabular}} & \multirow{2}{*}{$\begin{array}{c}\text { Cut } \\
\text { off }\end{array}$} & \multirow{2}{*}{$\begin{array}{l}\text { Sensi- } \\
\text { tivity }\end{array}$} & \multirow{2}{*}{$\begin{array}{l}\text { Speci- } \\
\text { ficity }\end{array}$} & \multirow{2}{*}{ PPV } & \multirow{2}{*}{ NPV } \\
\hline & & & $\mathbf{L L}$ & UL & & & & & \\
\hline IVCd $_{\max }$ & 0.746 & $<0.001$ & 0.687 & 0.805 & $\leq 1.84$ & 66.94 & 77.43 & 90.30 & 46.73 \\
\hline IVC CI & 0.894 & $<0.001$ & 0.857 & 0.931 & $>32.29$ & 84.50 & 89.09 & 97.7 & 66.36 \\
\hline
\end{tabular}

Table (9): Test Characteristics of the four ultrasound parameters in predicting fluid responsiveness at period of hypovolemia.

\begin{tabular}{|c|c|c|c|c|c|c|c|c|c|}
\hline \multirow{2}{*}{$\begin{array}{c}\begin{array}{c}\text { In presence } \\
\text { of signs of } \\
\text { hypovolemia }\end{array} \\
\end{array}$} & \multirow[b]{2}{*}{ AUC } & \multirow[b]{2}{*}{$\mathbf{p}$} & \multicolumn{2}{|c|}{ 95\% C.I } & \multirow{2}{*}{$\begin{array}{c}\text { Cut } \\
\text { off }\end{array}$} & \multirow{2}{*}{$\mid \begin{array}{l}\text { Sensi- } \\
\text { tivity }\end{array}$} & \multirow{2}{*}{$\begin{array}{l}\text { Speci- } \\
\text { ficity }\end{array}$} & \multirow{2}{*}{ PPV } & \multirow{2}{*}{ NPV } \\
\hline & & & $\mathbf{L L}$ & UL & & & & & \\
\hline $\mathbf{I V C d}_{\max }$ & 0.584 & 0.432 & 0.413 & .755 & $\leq 1.84$ & 80.29 & 39. & 75. & 5.05 \\
\hline IVC CI & 0.750 & $0.011 *$ & 0.606 & 0.89 & $>48.14$ & 86.47 & 52.5 & 81.6 & 9.96 \\
\hline
\end{tabular}




\section{DISCUSSION}

The primary aim of the present study was to evaluate the correlation between CVP measurements and ultrasound measurements of the maximum inferior vena cava diameter $\left(\mathrm{IVCd}_{\max }\right)$, collapsibility index (IVC CI). ${ }^{(12)}$ The secondary aim was to evaluate the value of ultrasound as a noninvasive tool in assessment of intravascular volume status and fluid responsiveness in intensive care unit patients. The present study was carried out on 50 adult patients aged 30- 60 years of either sex, ASA I-III, during which all patients were spontaneously breathing. The two ultrasound parameters were measured and their correlation with CVP measurements were investigated and statistically analyzed. This was performed at base line, immediately after bolus fluid administration, 3 and 6 hours after bolus fluid administration. The relation between CVP and ultrasound measurements at baseline was statistically analysed $(n=50)$. There was a significant negative correlation between CVP and IVC CI $(r=-0.788$, p $<0.001$ ), a significant positive correlation with $\operatorname{IVCd}_{\max }(\mathrm{r}=0.547, \mathrm{p}<0.001)$. Analysis of the ROC curve showed a better diagnostic accuracy of IVC CI (AUC 0.871, p <0.001) than $\mathrm{IVCd}_{\max }$, for predicting baseline low $\mathrm{CVP}<10 \mathrm{cmH}_{2} \mathrm{O}$, where IVC CI $>33.33 \%$ can predict low CVP with a sensitivity of $76.0 \%$ and a specificity of $92.0 \%$. In agreement with the present study, Zhang and Critchley ${ }^{(13)}$ assessed the relationship between $\mathrm{IVCd}_{\max }$ and IVC CI and the occurrence of hypotension. They found that ultrasound IVC measurements before bolus fluid administration were predictive of hypovolemia, and the CI was more predictive than IVCdmax $(\mathrm{P}=0.002)$. Cutoff values for predicting hypovolemia from IVC scanning were $43 \%$ for CI and $1.8 \mathrm{~cm}$ for IVCdmax. In 2016, Subramaniam et al. ${ }^{(14)}$ proposed that initial fluid optimization can perhaps be attempted with an IVC-CI target of $\leq 38 \%$ in an attempt to reduce the incidence of hypovolemia. Although they mentioned that this technique has the drawback that it prolongs the turn over time. The relation between CVP measurements and their corresponding measurements of the 2 ultrasound parameters in all the study periods was statistically analysed $(n=350)$ and showed similar results to the baseline results. In the present study, there was a significant negative correlation between CVP and IVC CI $(r=-0.788$, $\mathrm{p}<0.001$ ), where IVC CI $>32.17 \%$ can predict low CVP $<10 \mathrm{~cm} \mathrm{H}_{2} \mathrm{O}$ with a sensitivity of $80.48 \%$ and a specificity of $84.85 \%$. Many researchers have reported a good correlation between CVP and IVC respiratory variability in spontaneously breathing patients. In the study carried out by Kircher $\boldsymbol{e t}$ $\boldsymbol{a l}^{\left({ }^{(15)}\right.}$ where echocardiographic measurements were analyzed, the IVC CI greater than or equal to $50 \%$ indicated a CVP value below $10 \mathrm{~mm} \mathrm{Hg}$ and IVC CI below $50 \%$ indicated a CVP value of $10 \mathrm{~mm}$ $\mathrm{Hg}$ or more. In agreement with the present study, a study performed by Stawicki $\boldsymbol{e t}$ al. ${ }^{(16)}$ was done where intensivist instead of echocardiologist performed bedside ultrasonography examinations of the IVC. They concluded that collapsibility index IVC-CI examination can provide a useful guide to noninvasive volume status assessment in surgical ICU patients, and that IVC-CI appears to correlate best with CVP in the setting of low $(<0.20)$ and high $(>0.60)$ collapsibility ranges. Ilyas et al ${ }^{(17)}$ performed a cross-sectional study on 100 adult medical ICU patients for a period of 3 months. A patient with CVP of less than $8 \mathrm{~cm} \mathrm{H}_{2} \mathrm{O}$ was considered as hypovolemic. Patients with CVP between 8-12 $\mathrm{cm} \quad \mathrm{H}_{2} \mathrm{O}$ were considered as euvolemic and patients having CVP $>12 \mathrm{~cm} \mathrm{H}_{2} \mathrm{O}$ were considered as hypervolemic. There was a strong negative linear correlation between the CVP $\left(10.38 \pm 4.14 \mathrm{~cm} \mathrm{H}_{2} \mathrm{O}\right)$ and the IVC CI (\%) (30.68 $\pm 10.93)$, which was statistically significant. In a prospective double-blind observational study conducted by Worapratya $\boldsymbol{e t} \boldsymbol{a l}{ }^{(18)}$ in the emergency room of a tertiary care center. All patients who presented with shock and had a central venous catheter insertion performed were enrolled. The correlation of CVP and the caval index were calculated by Pearson's correlation coefficient. The participants' characteristics were divided into CVP $<10 \mathrm{~cm} \mathrm{H}_{2} \mathrm{O}, 10-15 \mathrm{~cm} \mathrm{H}_{2} \mathrm{O}$ and $>15 \mathrm{~cm} \mathrm{H}_{2} \mathrm{O}$. The correlation coefficient of the CVP measurement with the ultrasound IVC caval index was $r=-0.647(\mathrm{P}=0.001)$. The sensitivity and specificity of the caval index were calculated to predict the CVP. The results showed that the cutoff points of the caval index were 30,20, and 10 at CVP levels $<10 \mathrm{~cm} \mathrm{H}_{2} \mathrm{O}, 10-15 \mathrm{~cm} \mathrm{H}_{2} \mathrm{O}$ and $>15$ $\mathrm{cm} \mathrm{H}_{2} \mathrm{O}$, respectively. The ultrasonographic parameter measured in the present study, other than the collapsibility index, showed a positive correlation with CVP measurements. In the present study, there was a significant positive 
correlation between CVP and $\operatorname{IVCd}_{\max }(\mathrm{r}=$ $0.547, \mathrm{p}<0.001)$ where $\mathrm{IVCd}_{\max } \leq 1.73 \mathrm{~cm}$ can predict low CVP $<10 \mathrm{~cm} \mathrm{H}_{2} \mathrm{O}$ with a sensitivity of $71.4 \%$ and a specificity of $75.6 \%$. Several other studies were performed to investigate the best ultrasound parameter that correlates well with the CVP. Worapratya et al. ${ }^{(18)}$ performed a correlation study between CVP and IVC end expiratory diameter and on 25 spontaneously breathing patients. Co-morbidities included diabetes mellitus $(80 \%)$, hypertension $(68 \%)$, ischemic heart disease (24\%), chronic kidney disease (20\%). Sepsis was the primary diagnosis in nearly $80 \%$ of the patients. $60 \%$ cases had had central venous access to guide fluid therapy; in $40 \%$ it was for infusion of a vasoactive agent. Five patients (20\%) were obese. They concluded that bedside ultrasound measurements of the IVC at end expiration correlates well with CVP measurements using central venous access. Similar to the present study, Nagdev et $\boldsymbol{a l}{ }^{\left({ }^{(19)}\right.}$ also performed a prospective observational study on adult patients undergoing central venous catheterization. Inferior vena cava inspiratory and expiratory diameters were measured by 2-dimensional bedside ultrasonography. The correlation of central venous pressure and caval index was calculated. The sensitivity, specificity, and positive and negative predictive values of a caval index greater than or equal to $50 \%$ that was associated with a central venous pressure less than $8 \mathrm{~mm} \mathrm{Hg}$ were estimated. Their correlations of CVP measurement with the ultrasonographic measurements were, respectively, expiratory inferior vena cava diameter (r 0.66), and caval index $(\mathrm{r}-0.74)$. In contrast to this study, Prekker et al $^{(20)}$ studied the correlation between CVP and ultrasound measurements of IVC maximum diameter, IVC CI to width ratio in 67 spontaneously breathing mostly septic ICU patients. They found that the area under the ROC curve to discriminate a low central venous pressure (< $10 \mathrm{~mm} \mathrm{Hg}$ ) was 0.91 for inferior vena cava diameter, which was significantly higher than the IVC CI 0.66. An inferior vena cava diameter $<2$ $\mathrm{cm}$ predicted a central venous pressure $<10 \mathrm{~mm}$ $\mathrm{Hg}$ with a sensitivity of $85 \%$, specificity of $81 \%$. Prekker et al. ${ }^{(20)}$ took cutoffs of IVC diameter $<2$ $\mathrm{cm}$, IVC CI $>50 \%$, in predicting a CVP $<10 \mathrm{~mm}$ $\mathrm{Hg}$. This contradiction to the present study might be attributed to different cutoff points from this study. They also performed their study on ICU patients admitted due to sepsis which may have affected the patients' systemic vascular resistance and possibility of presence of both regional and global abnormalities of left ventricular function in patients with sepsis. ${ }^{(21)}$ Also some of their patients had respiratory failure which might have affected patient's respiratory efforts and therefore affected the IVC collapsibility with inspiration. While in the present study, measurements were performed, on healthy individuals after exclusion of such coexisting diseases. A study by Blehar et al. ${ }^{(22)}$ in acutely dyspneic patients presenting to the emergency department showed that IVC sonography rapidly identifies patients with congestive heart failure and volume overload. The IVC was measured sonographically during a complete respiratory cycle of 46 patients meeting study criteria. Percentage of respiratory variation of IVC diameter was compared to the diagnosis of $\mathrm{CHF}$ or alternative diagnosis. Respiratory variation of IVC was less in patients with CHF (9.6\%) than without CHF (46\%) and showed good diagnostic accuracy with area under the ROC curve of 0.96 . Receiver operating characteristic curve analysis showed optimum cutoff of $15 \%$ variation or less of IVC diameter with $92 \%$ sensitivity and $84 \%$ specificity for the diagnosis of $\mathrm{CHF}$. The relation between CVP measurements and their corresponding measurements of the 2 ultrasound parameters during hypovolemia was statistically analyzed $(n=50)$ showed that there was a significant negative correlation between CVP and IVC CI $(\mathrm{r}=-0.364, \mathrm{p}=0.009)$ and a significant positive correlation between CVP and $\mathrm{IVCd}_{\max }(\mathrm{r}=0.262, \mathrm{p}=0.066)$. After administration of $6 \%$ hydroxyethyl starch (HES, 130/0.4) fluid bolus of $7 \mathrm{ml} / \mathrm{kg}$, there was a significant increase in MABP, CVP, $\operatorname{IVCd}_{\max }$, while there was a significant decrease in IVC CI $(\mathrm{p}<0.001)$. A study by Ferrada et al ${ }^{(23)}$ in trauma patients showed the value of bedside caval sonography in evaluation of fluid status and resuscitation of critically ill patients. Prospective evaluation of hemodynamic status was performed in hypotensive patients via limited transthoracic echocardiogram (LTTE). Qualitative assessment of the IVC was obtained via subxiphoid window. FLAT IVC was defined as diameter less than $2 \mathrm{~cm}$ and FAT IVC when the vein was equal to or larger than $2 \mathrm{~cm}$. Collapsibility was assessed by observing respiratory variation of the vessel. 
Lactate was measured before and after therapy was initiated. A follow-up LTTE was obtained after fluid challenge. A total of 108 LTTE were performed. Sixty-nine patients were mechanically ventilated at time of LTTE. Seventy-three patients had a FLAT IVC, and received fluid challenge as therapy. All patients had a change in IVC volume from "FLAT" to "FAT" after the fluid challenge. Seventy-one patients $(97 \%)$ had resolution of hypotension after the first fluid challenge. Two patients had persistent hypotension and received a second fluid challenge. Follow-up LTTE demonstrated a FAT IVC and lack of collapsibility. Lactate decreased in all 73 patients after therapy guided by LTTE. Another prospective observational study was performed by Weekes $\boldsymbol{e t}$ $\boldsymbol{a l} .^{(24)}$ in emergency department hypotensive patients. Patients were eligible for enrollment if they were hypotensive as defined by a systolic blood pressure (sBP) of $<100 \mathrm{~mm} \mathrm{Hg}$ or mean arterial pressure of $\leq 65 \mathrm{~mm} \mathrm{Hg}$, exhibited signs or symptoms of shock, and the treating physician intended to administer intravenous (IV) fluid boluses up to $20 \mathrm{ml} / \mathrm{kg}$ for resuscitation. Sonologists performed a sequence of echocardiographic assessments at the beginning, during, and toward the end of fluid challenge. Both caval index and left ventricle systolic function (LVF) were determined by the sonologist in qualitative then quantitative manners. The IVC CI was shown to decline after volume expansion in patients in hypovolemic shock. They concluded that the use of IVC CI during fluid resuscitation correlated well with LVF. Yanagawa et $\boldsymbol{a l} .{ }^{(25)}$ measured IVC diameters in 35 trauma patients, with 10 of them in shock and 25 in a stable hemodynamic state, on arrival to the emergency department and again at day 5. They found significantly smaller IVC diameters in the group of patients in shock on arrival in comparison with those not in shock. The same group of investigators demonstrated that the IVC diameter is useful in predicting the response of patients in shock states to fluid resuscitation. In this study, the IVC diameter at the end of expiration was measured in trauma patients with hemorrhagic shock at baseline and again after what was believed to be adequate fluid resuscitation (defined by the improvement of systolic blood pressure to a level greater than 90 $\mathrm{mm} \mathrm{Hg}$ ). Individuals who were able to maintain a stable blood pressure after fluid resuscitation had a significant increase in end-expiratory IVC diameter, whereas those who remained hemodynamically unstable did not have a change in IVC diameter with resuscitation. The authors concluded that changes in IVC diameter in response to fluid resuscitation is a better indicator of adequate fluid resuscitation than vital signs. Sefidbakht et $\boldsymbol{a l l}^{\left({ }^{(26)}\right.}$ also demonstrated significantly smaller IVC diameters at the end of expiration or inspiration in a group of patients in shock as compared with controls (5.6 and $4.0 \mathrm{~mm}$ vs. 11.9 and $9.6 \mathrm{~mm}$, respectively). In addition, they showed a higher vena cava collapsibility (or pulsatility) index in the shock group. Authors of the study demonstrated that measurement of IVC diameter may be a reliable indicator of shock in trauma patients and may even predict it in patients who still have normal blood pressure due to sympathetic overactivity. In their study, one subject with a small IVC diameter in the shock group showed normal values for both blood pressure and pulse rate at arrival, but these values deteriorated within $12 \mathrm{~h}$ of arrival. They also mentioned that serial measurements of IVC diameter can be used to monitor ongoing blood loss and monitor fluid therapy and can even be used as an alternative to direct central venous pressure measurement, which is not suitable as a routine procedure. As regards prediction of fluid responsiveness, a patient was defined as fluid responder if the mean arterial pressure increased by at least $20 \%$ as compared with pre-fluid challenge values. Several studies also used MABP as a guide for fluid administration, and defined fluid responsiveness by the increase in MABP. ${ }^{(27)}$ There was 34 responders $(68 \%)$ and 16 non-responders (32\%) after fluid challenge. The non-responders needed further repeated fluid boluses administration as long as there is positive response and until improvement of the pre-fluid challenge sign of hypovolemia. Analysis of the ROC curve showed a better diagnostic accuracy of IVC collapsibility index and IVC diameter, for predicting fluid responsiveness, where IVC CI $>48.14 \%$ can predict fluid responsiveness with a sensitivity of $86.47 \%$ and a specificity of $52.50 \%$, a positive predictive value of $77.8 \%$ and a negative predictive value of $57.1 \%$. The area under curve AUC for IVC CI was 0.735 with $\mathrm{p} 0.008$, which was significant. In agreement with the present study, several studies have suggested that assessment of IVC collapsibility is 
useful in the critically ill patient whose caval index approaches the extremes. Additionally, caval sonography can be repeated during resuscitation to evaluate improvement of these parameters. Muller et $\boldsymbol{a l} .{ }^{(28)}$ in their study on spontaneously breathing patients with acute circulatory failure concluded that despite its apparent simplicity, IVC collapsibility index should be interpreted with caution. A high IVC CI value (> 40\%) is usually associated with fluid responsiveness while low values $(<40 \%)$ do not exclude fluid responsiveness. Several previous studies reported the importance of point of care ultrasound in the management plan of patients with sepsis. In the study by Haydar $\boldsymbol{e t}$ al. ${ }^{(29)}$ patients with suspected sepsis received point-of-care ultrasonography to determine cardiac contractility, inferior vena cava diameter, and inferior vena cava collapsibility. Physician reports of treatment plans, presumed causes of observed vital sign abnormalities, and degree of certainty were compared before and after knowledge of point-of-care ultrasonographic findings. Seventy-four adult sepsis patients were enrolled: 27 (37\%) sepsis, $30(40 \%)$ severe sepsis, $16(22 \%)$ septic shock, and $1(1 \%)$ systemic inflammatory response syndrome. After receipt of point-of-care ultrasonographic data, physicians altered the presumed primary cause of vital sign abnormalities in 12 cases and procedural intervention plans in 20 cases. Overall treatment plans were changed in 39 cases. Certainty increased in $47(71 \%)$ cases and decreased in 19 (29\%). They concluded that point-of-care sonography evaluating cardiac contractility and IVC collapsibility in patients with suspected sepsis was shown to increase physician certainty and alter more than $50 \%$ of treatment plans. When compared with the dynamic parameters for assessment of fluid responsiveness, Machare-Delgado et $\boldsymbol{a l} .{ }^{(30)}$ compared IVC change to pulse contour analysis in an ICU population after a $500-\mathrm{mL}$ bolus and demonstrated much better correlation between stroke volume improvement and IVC change than with stroke volume variation in pulse contour analysis, but patients in this study also were mechanically ventilated. This study had some limitations. First, there is variation of patients' respiratory rate and effort. Second limitation is that ultrasound is an operator dependent procedure and requires specific training skills to avoid any technical errors in measurement.

\section{CONCLUSION}

Ultrasound of the inferior vena cava may be used as a feasible non-invasive, rapid and simple adjuvant method to assess the intravascular volume and guide fluid responsiveness in critically ill intensive care unit patients. Inferior vena cava collapsibility index may be used to predict low central venous pressure and predict fluid responsiveness.

\section{CONFLICTS OF INTEREST}

There are no conflicts of interest.

\section{REFERENCES}

1- The National Heart, Lung, and Blood Institute Acute Respiratory Distress Syndrome (ARDS) (2006): Clinical Trials Network. Comparison of two fluidmanagement strategies in acute lung injury. N Engl J Med., 354:2564-2575.

2- Marik PE (2011): of Evidence-Based Critical Care, 55. DOI 10.1007/978-14419-5923-2_8.

3- Ruesch S, Walder B, Tramer MR (2002): Complications of central venous catheters: Internal jugular versus subclavian access - A systematic review. Crit Care Med.., 30:454-460.

4- Gray's anatomy (2008): the anatomical basis of clinical practice (40th ed.). London: Churchill Livingstone.

5- Durairaj L, Schmidt GA (2008): Fluid therapy in resuscitated sepsis: Less is more. Chest, 133: 252-63.

\section{6- Demeria DD, MacDougall A, Spurek M} (2004): Comparison of clinical measurement of jugular venous pressure versus measured central venous pressure. Chest, 126: 747.

7- Cherpanath TG, Aarts LP, Groeneveld JA, Geerts BF (2014): Defining fluid responsiveness: a guide to patient-tailored volume titration. J Cardiothorac Vasc Anesth., 28(3):745-54. 
8- Sridhar H, Mangalore P, Chandrasekaran vp, Manikam R (2012): Caval Aorta Index and Central Venous Pressure Correlation in Assessing Fluid Status! "Ultrasound Bridging the Gap". International Scholarly Research Network ISRN Emergency Medicine, 1-5.

9- Seif D, Mailhot T, Perera $P$, Mandavia $D$ (2012): Caval sonography in shock: a noninvasive method for evaluating intravascular volume in critically ill patients. J Ultrasound Med., 31(12):1885-90.

10- Lakhal K, Ehrmann S, Runge I, Benzekri-Lefevre D, Legras A, Dequin PF et al. (2016): Central venous pressure measurements improve the accuracy of leg raising-induced change in pulse pressure to predict fluid responsiveness. Intensive Care Med., 36(6):940-8.

11- Natalini G, Rosano A, Militano CR, Di Maio A, Ferretti P, Bertelli $M$ et al. (2012): Prediction of arterial pressure increase after fluid challenge. BMC Anesthesiol., 12:3.

12- Donahue SP, Wood JP, Patel BM, Quinn JV (2009): Correlation of sonographic measurements of the internal jugular vein with central venous pressure. Am J Emerg Med., 27(7):851-5.

13- Zhang J, Critchley LA (2016): Inferior Vena Cava Ultrasonography before General Anesthesia Can Predict Hypotension after Induction. Anesthesiology, 124(3):580-9.

14- Subramaniam B, Subramaniam K (2016): Cautious Optimism: Can Preoperative Ultrasound Predict Postinduction Hypotension? Anesthesiology, 124(3):526-7.

15- Kircher BJ, Himelman RB, Schiller NB (1990): Noninvasive estimation of right atrial pressure from the inspiratory collapse of the inferior vena cava. Am J Cardiol., 66(4):493-6.

16- Stawicki SP, Braslow BM, Panebianco NL, Kirkpatrick JN, Gracias VH, Hayden GE et al. (2009): Intensivist use of handcarried ultrasonography to measure IVC collapsibility in estimating intravascular volume status: correlations with CVP. J Am Coll Surg., 209(1):55-61.
17- Ilyas A, Ishtiaq W, Assad S, Ghazanfar H, Mansoor S, Haris M et al. (2017): Correlation of IVC Diameter and Collapsibility Index With Central Venous Pressure in the Assessment of Intravascular Volume in Critically Ill Patients. Cureus,9(2):e1025.

18- Worapratya P, Anupat S, Suwannanon R, Wuthisuthimethawee $P$ (2014): Correlation of caval index, inferior vena cava diameter, and central venous pressure in shock patients in the emergency room. Open Access Emerg Med., 6:57-62.

19- Nagdev AD, Merchant RC, TiradoGonzalez A, Sisson CA, Murphy MC (2010): Emergency department bedside ultrasonographic measurement of the caval index for noninvasive determination of low central venous pressure. Ann Emerg Med., 55(3):290-5.

20- Prekker ME, Scott NL, Hart D, Sprenkle MD, Leatherman JW (2013): Point-ofcare ultrasound to estimate central venous pressure: a comparison of three techniques. Crit Care Med., 41(3):833-41.

21- Young JD (2004): The heart and circulation in severe sepsis. $\mathrm{Br} \mathrm{J}$ Anaesth., 93(1):114-20.

22- Blehar DJ, Dickman E, Gaspari R (2009): Identification of congestive heart failure via respiratory variation of inferior vena cava diameter. Am J Emerg Med., 27(1):71-5.

23- Ferrada P, Anand RJ, Whelan J, Aboutanos MA, Duane T, Malhotra A et al. (2012): Qualitative assessment of the inferior vena cava: useful tool for the evaluation of fluid status in critically ill patients. Am Surg., 78(4):468-70.

24- Weekes AJ, Tassone HM, Babcock A, Quirke DP, Norton HJ, Jayarama K et al. (2011): Comparison of serial qualitative and quantitative assessments of caval index and left ventricular systolic function during early fluid resuscitation of hypotensive emergency department patients. Acad Emerg Med., 18(9):91221. 
25- Yanagawa Y, Sakamoto T, Okada Y (2007): Hypovolemic shock evaluated by sonographic measurement of the inferior vena cava during resuscitation in trauma patients. J Trauma, 63(6):1245-8.

26- Sefidbakht S, Assadsangabi R, Abbasi HR, Nabavizadeh A (2007): Sonographic measurement of the inferior vena cava as a predictor of shock in trauma patients. Emerg Radiol., 14(3):181-5.

27- Cecconi M, Parsons AK, Rhodes A (2011): What is a fluid challenge? Curr Opin Crit Care, 17(3):290-5.

28- Muller L, Bobbia X, Toumi M, Louart G, Molinari N, Ragonnet B et al. (2012): Respiratory variations of inferior vena cava diameter to predict fluid responsiveness in spontaneously breathing patients with acute circulatory failure: need for a cautious use. Crit Care, 16(5):R188.
29- Haydar SA, Moore ET, Higgins GL, Irish CB, Owens WB, Strout TD (2012):

Effect of bedside ultrasonography on the certainty of physician clinical decisionmaking for septic patients in the emergency department. Ann Emerg Med., 60(3):346-58 e4.

30- Machare-Delgado E, Decaro M, Marik PE (2011): Inferior vena cava variation compared to pulse contour analysis as predictors of fluid responsiveness: a prospective cohort study. J Intensive Care Med., 26(2):116-24. 